\title{
SYNTHESIS AND ANTIMICROBIAL ACTIVITIES OF BENZOTHIOPHENE DERIVATIVES
}

\author{
GADADA NAGANAGOWDA, PATCHANITA THAMYONGKIT, AMORN PETSOM*
}

Research Center for Bioorganic Chemistry, Department of Chemistry, Faculty of Science, Chulalongkorn University, Bangkok 10330, Thailand:

(Received: August 5, 2011 - Accepted: January 10, 2012)

\begin{abstract}
3-Chlorobenzothiophene-2-carbonyl chloride $\mathbf{1}$ was reacted with 4-aminoacetophenone in acetone to give compound 2. In order to prepare chalcones 3a-d, compound $\mathbf{2}$ was condensed with various aldehydes in the presence of $\mathrm{KOH}$ in DMF. These chalcones 3a-d on cyclization with urea, thiourea, hydroxylamine hydrochloride, phenyl hydrazines and hydrazine hydrate gave the corresponding oxapyrimidines 4a-d, isoxazolines 5a-d, pyrazoles 6a-h, pyrazolines 7a-d and thiopyrimidines 8a-d, respectively. The structures of all the synthesized compounds were confirmed by spectral data and had been screened for antibacterial activity.
\end{abstract}

Keywords: Benzothiophene, isoxazolines, pyrimidines, pyrazolines, antimicrobial activity

\section{INTRODUCTION}

Chalcones are $\alpha, \beta$-unsaturated ketones containing a reactive ketoethylenic - $\mathrm{CO}-\mathrm{CH}=\mathrm{CH}-$ group. The presence of $\alpha, \beta$-unsaturated carbonyl system in the chalcone nucleus makes it biologically active. Some substituted chalcones and their derivatives have been reported to possess some interesting biological properties such as antifungal ${ }^{1}$, insecticidal ${ }^{2}$, anaesthetic ${ }^{3}$ and ulcerogenic ${ }^{4}$ activities. In addition, chalcones serve as intermediates for the synthesis of various heterocycles such as pyrazolones, oxazoles, pyrimidines etc., which are found to have extensive pharmaceutical applications. Oxapyrimidines and thiopyrimidines are currently used in the chemotherapy of Acquired Immune Deficiency Syndrome (AIDS). Several pyrimidine derivatives containing drugs have exhibited antiulcer ${ }^{5}$ and anti-AIDS ${ }^{6}$ activities. The pyrimidine nucleus occurs in biologically important products such as nucleic acids, vitamins, coenzymes and pharmacologically useful products of plant origin. With the intention to synthesize more potent antimicrobial agents, the pyrimidine moiety has been condensed with different types of heterocycles such as furan', thiophene ${ }^{8}$, pyrrole ${ }^{9}$, pyrazole ${ }^{10-11}$, thiazole ${ }^{12}$, imidazole ${ }^{13-14}$, pyrazine ${ }^{15}$ and indole ${ }^{16}$ etc.

Further to our search for new antimicrobial agents we report herein the preparation of new isoxazoline, pyrimidine and pyrazoline chalcone derivatives from 4-acetylphenyl-3-chloro-1-benzothiophene-2-carboxamide compound. The antibacterial and antifungal activities of the resulting derivatives were screened and the relationship of molecular structure and the bioactivity are discussed.

\section{RESULTS AND DISCUSSION}

The preparation of the target chalcones 3a-d started from the amidation of 3-chlorobenzothiophene-2-carbonyl chloride 1 , which was prepared by the reaction of cinnamic acid with thionyl chloride in DMF and pyridine according to the reported method ${ }^{17}$. Upon condensation of compound 2 and various aldehydes in the presence of $\mathrm{KOH}$ in DMF, chalcones 3a-d were obtained in $80-86 \%$ yield (Scheme-1). Further condensation of chalcones 3a-d with urea, thiourea, hydroxylamine hydrochloride, hydrazine hydrate and various phenylhydrazines, respectively, oxo-pyrimidines $4 \mathbf{a}-\mathbf{d}$, thio-pyrimidines 8ad, isoxazolines 5a-d, pyrazolines 7a-d and pyrazoles $\mathbf{6 a - h}$ were successfully prepared. (Scheme-2).

\section{ANTIMICROBIAL EVALUATION \\ 3.1. Antibacterial activity}

A Cup plate method using Hi-Media agar medium was employed to study the antibacterial activity of the synthesized compounds against two Grampositive bacteria, Staphylococcus aureus-ATCC 25923 and Bacillus subtilisATCC 6633 and Gram-negative bacteria, Pseudomonas aeruginosa-ATCC 10145 and Escherichia coli-ATCC 35218. Preparation of nutrient broth, subculture, base layer medium, agar medium and peptone water was done as per the standard procedure ${ }^{18}$. The results of the study are summarized in Table-1. The tested compound showed slight to moderate antibacterial activity compared to the standard drugs against all microorganisms.
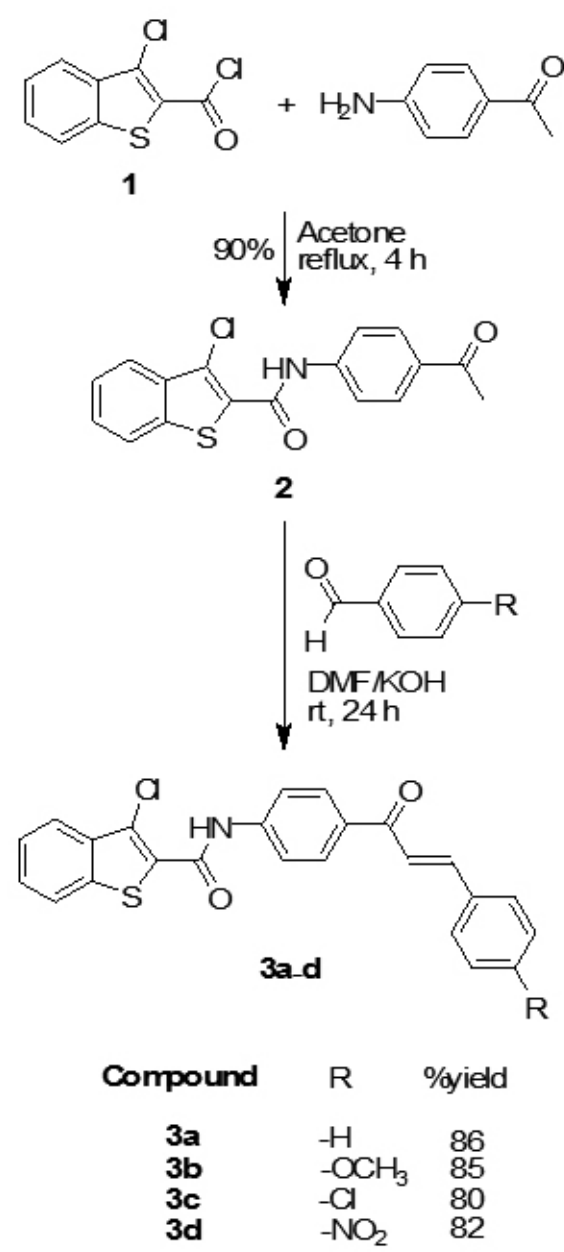

Scheme-1: Synthesis of chalcones 3a-d

\subsection{Antifungal activity}

The antifungal activity of the synthesized compounds was tested against four different fungi, i.e. Candida albicans, Crysosporium pannical, Aspergillus niger and Rhizopus oryzae by a filter paper disc technique ${ }^{19}$. The concentration of test compounds was $1000 \mu \mathrm{g} / \mathrm{mL}$. After $48 \mathrm{~h}$ treatment, zone of inhibition produced by each compound was measured in $\mathrm{mm}$. Griseofulvin was used as the standard antifungal agent and dimethyl formamide as a control. The results are described in Table-2. 


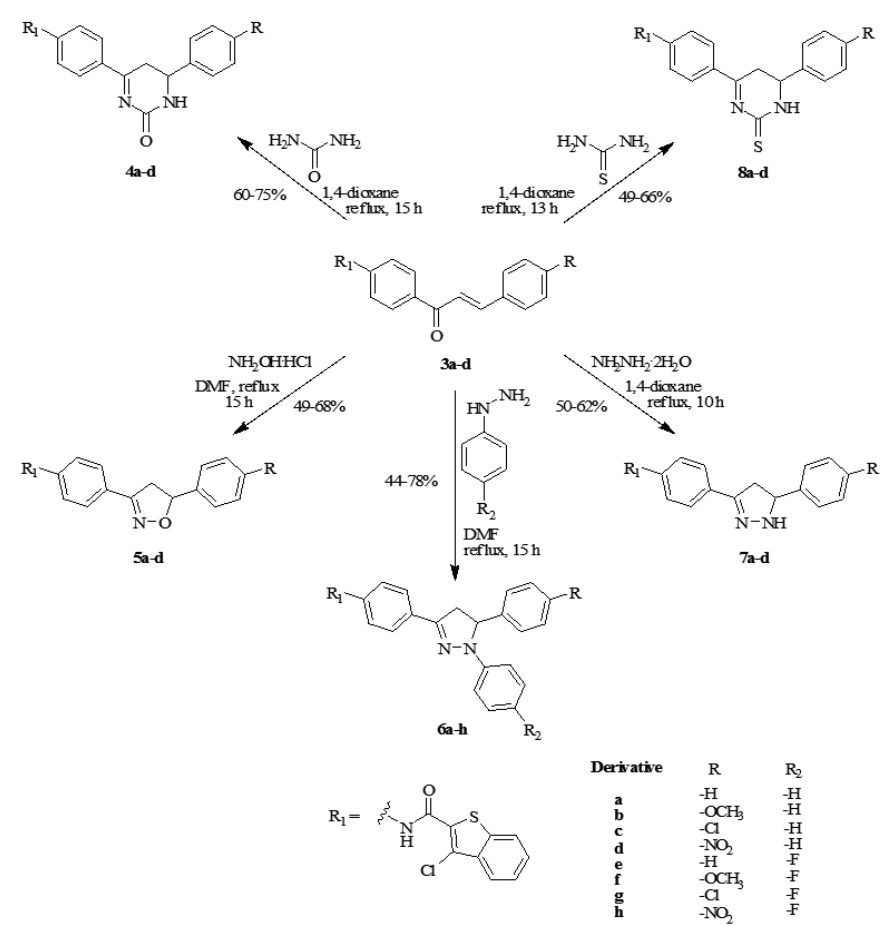

Scheme-2: General synthetic procedure for oxo-pyrimidines 4a-d, isoxazolines 5a-d, pyrazoles 6a-h, pyrazolines 7a-d and thio-pyrimidines 8a-d.

Table-1. Antibacterial activity of the tested compounds.

\begin{tabular}{|c|c|c|c|c|}
\hline \multirow{2}{*}{ Compound } & \multicolumn{4}{|c|}{ Zone of inhibition (mm) } \\
\cline { 2 - 5 } & Gram positive bacteria & \multicolumn{2}{c|}{ Gram negative bacteria } \\
\cline { 2 - 5 } & S. aureus & B. subtilis & $\begin{array}{c}\text { P. } \\
\text { aeruginosa }\end{array}$ & E. coli \\
\hline $\mathbf{2}$ & 11 & 14 & 13 & 14 \\
\hline 3a & 14 & 12 & 15 & 12 \\
\hline 3b & 11 & 16 & 14 & 15 \\
\hline 4a & 13 & 11 & 14 & 10 \\
\hline 4b & 14 & 11 & 14 & 13 \\
\hline 5a & 10 & 14 & 13 & 13 \\
\hline $\mathbf{5 b}$ & 13 & 15 & 14 & 12 \\
\hline $\mathbf{6 a}$ & 11 & 14 & 14 & 13 \\
\hline $\mathbf{6 b}$ & 13 & 12 & 14 & 13 \\
\hline $\mathbf{6 e}$ & 13 & 16 & 12 & 11 \\
\hline 7a & 14 & 12 & 15 & 13 \\
\hline 7b & 15 & 13 & 14 & 13 \\
\hline $\mathbf{8 a}$ & 10 & 14 & 10 & 12 \\
\hline $\mathbf{8 b}$ & 12 & 16 & 10 & 14 \\
\hline Control(DMF) & 00 & 00 & 00 & 00 \\
\hline Ampicillin & 23 & 24 & 23 & 18 \\
\hline Streptomycin & 24 & 22 & 21 & 25 \\
\hline & & & & \\
\hline
\end{tabular}

Table-2. Antifungal activity of the tested compounds.

\begin{tabular}{|c|c|c|c|c|}
\hline \multirow{2}{*}{ Compound } & \multicolumn{4}{|c|}{ Zone of inhibition (mm) } \\
\cline { 2 - 5 } & C. albicans & $\begin{array}{c}\boldsymbol{C} \text {. } \\
\text { pannical }\end{array}$ & A.niger & R. oryzae \\
\hline $\mathbf{2}$ & 14 & 11 & 13 & 13 \\
\hline 3a & 14 & 13 & 14 & 12 \\
\hline 3b & 13 & 14 & 13 & 11 \\
\hline 4a & 12 & 12 & 12 & 14 \\
\hline 4b & 15 & 15 & 14 & 15 \\
\hline 5a & 15 & 14 & 15 & 11 \\
\hline 5b & 13 & 16 & 14 & 12 \\
\hline $\mathbf{6 a}$ & 15 & 15 & 13 & 11 \\
\hline $\mathbf{6 b}$ & 14 & 13 & 13 & 11 \\
\hline 6e & 15 & 12 & 12 & 15 \\
\hline 7a & 16 & 12 & 13 & 12 \\
\hline $7 b$ & 12 & 15 & 11 & 14 \\
\hline 8a & 14 & 14 & 15 & 15 \\
\hline $\mathbf{8 b}$ & 14 & 13 & 13 & 12 \\
\hline Control(DMF) & 00 & 00 & 00 & 00 \\
\hline Griseofulvin & 24 & 25 & 23 & 22 \\
\hline & & & & \\
\hline
\end{tabular}

\section{EXPERIMENTAL}

All chemicals were analytical grade, purchased from commercial suppliers and used as received without further purification. Melting points were determined in open capillary and were uncorrected. FT-IR spectra were recorded on a Nicolet Fourier Transform Infrared Spectrophotometer: Impact 410 (Nicolet Instrument Technologies, Inc. WI, USA). Infrared spectra were recorded between $400 \mathrm{~cm}^{-1}$ to $4,000 \mathrm{~cm}^{-1}$ in transmittance mode. ${ }^{1} \mathrm{H}-\mathrm{NMR}$ and ${ }^{13} \mathrm{C}$-NMR were obtained in DMSO- $d_{6}$ at $400 \mathrm{MHz}$ for ${ }^{1} \mathrm{H}$ nuclei and $100 \mathrm{MHz}$ for ${ }^{13} \mathrm{C}$ nuclei (Varian Company, USA). All chemical shifts were reported in parts per million (ppm) using residual proton or carbon signal in deuterated solvents as internal references. Mass spectra were obtained using matrixassisted laser desorption ionization mass spectrometry (MALDI-TOF) by using dithranol as a matrix. Elemental analysis $(\mathrm{C}, \mathrm{H}, \mathrm{N}$ and $\mathrm{S})$ was performed on Perkin Elmer 2400 analyzer. The purity of the compound was checked by TLC on silica gel and further purification was performed through column chromatography (silica gel, 60-120 mesh).

\subsection{Preparation of 3-chloro-1-benzothiophene-2-carbonylchloride (1)}

The compound 1 was prepared according to the literature procedure ${ }^{17} \mathrm{~m} . \mathrm{p}$ $112-114^{\circ} \mathrm{C}$ (Lit.mp $\left.110-112^{\circ} \mathrm{C}\right)$

\subsection{Preparation of 4-(acetylphenyl)-3-chloro-1-benzothiophene-2-} carboxamide (2)

A mixture of 4-aminoacetophenone $(1.35 \mathrm{~g}, 0.01 \mathrm{~mol})$ and 3-chloro-1benzothiophene-2-carbonylchloride $(2.31 \mathrm{~g}, 0.01 \mathrm{~mol})$ was dissolved in dry acetone $(40 \mathrm{~mL})$. The reaction mixture was refluxed for $4 \mathrm{~h}$. Periodically, sodium carbonate was added to neutralize $\mathrm{HCl}$ evolved during the reaction. Finally, the reaction mixture was cooled and poured into crushed ice. The resulting precipitate was filtered, washed with water, dried and recrystallized from methanol to give compound $\mathbf{2}$ as white needles.

IR $v\left(\mathrm{~cm}^{-1}\right): 3220(\mathrm{~N}-\mathrm{H}), 1650(\mathrm{C}=\mathrm{O}), 1562(\mathrm{C}=\mathrm{C}), 1080(=\mathrm{C}-\mathrm{Cl}), 688$ (C-S-C); ${ }^{1} \mathrm{H}-\mathrm{NMR} \delta$ (ppm): 10.89 (s, $\left.1 \mathrm{H}, \mathrm{CONH}\right), 8.19-7.60$ (m, 8H, Ar-H), $2.55\left(\mathrm{~s}, 3 \mathrm{H}, \mathrm{CH}_{3}\right) ;{ }^{13} \mathrm{C}-\mathrm{NMR} \delta$ (ppm): 197.0, 161.8, 142.3, 141.6, 136.6, 135.9, 133.4, 129.9, 129.0, 126.7, 124.4, 124.3, 122.8, 121.5, 26.6; MS: m/z, 329.80 $\left(\mathrm{M}^{+}\right)$. Anal. calcd. for $\mathrm{C}_{17} \mathrm{H}_{12} \mathrm{ClNO}_{2} \mathrm{~S}$ : C, 61.91; H, 3.67; N, 4.25; S, 9.72; found: $\mathrm{C}, 61.87 ; \mathrm{H}, 3.62 ; \mathrm{N}, 4.21 ; \mathrm{S}, 9.71 \%$.

\subsection{General procedure for synthesis of compounds $3 a-d$.}

Exemplary detail for 3-chloro- $N-\{4-[(2 E)-3-p h e n y l p r o p-2-e n o y l]$ 
phenyl\}-1-benzothiophene-2-carboxamide (3a)

N-(4-Acetylphenyl)-3-chloro-1-benzothiophene-2-carboxamide 2 (3.29 g, $0.01 \mathrm{~mol})$ was dissolved in DMF $(20 \mathrm{~mL})$ and benzaldehyde $(1.06 \mathrm{~g}, 0.01 \mathrm{~mol})$ was added to the reaction mixture with constant stirring at room temperature. Then $40 \% \mathrm{KOH}$ in distilled water was added to the reaction mixture with constant stirring at room temperature. After $24 \mathrm{~h}$, the reaction mixture was poured into crushed ice and neutralized with $\mathrm{HCl}$. The precipitate was filtered, washed with water, dried and recrystallized from methanol. The resulting solid was further purified by column chromatography using a gradient mixture of n-hexane and chloroform (90:10) as an eluent to obtain 3a. Compounds 3b-d were prepared by similar methodology.

IR $v\left(\mathrm{~cm}^{-1}\right): 3223(\mathrm{~N}-\mathrm{H}), 1655(\mathrm{C}=\mathrm{O}) ;{ }^{1} \mathrm{H}-\mathrm{NMR} \delta(\mathrm{ppm}): 9.14(\mathrm{~s}, 1 \mathrm{H}$, $\mathrm{CONH}), 8.13-7.26(\mathrm{~m}, 13 \mathrm{H}, \mathrm{Ar}-\mathrm{H}), 6.74-6.52(\mathrm{~d}, 2 \mathrm{H}, \mathrm{CH}=\mathrm{CH}) ;{ }^{13} \mathrm{C}-\mathrm{NMR} \delta$ (ppm): 189.7, 161.7, 145.1, 143.7, 141.6, 135.9, 135.3, 133.4, 133.1, 129.9, $128.6,128.5,127.9,126.7,124.4,124.3,122.8,122.3,121.1 ; \mathrm{MS}: \mathrm{m} / \mathrm{z}, 417.90$ $\left(\mathrm{M}^{+}\right)$. Anal. calcd. for $\mathrm{C}_{24} \mathrm{H}_{16} \mathrm{ClNO}_{2} \mathrm{~S}$ : C, 68.98; H, 3.86; N, 3.35; S, 7.67; found: C, 68.87; H, 3.83; N, 3.30; S, 7.62\%.

4.3.1. 3-Chloro-N-\{4-[(2E)-3-(4-methoxyphenyl)prop-2-enoyl]phenyl\}-1benzothiophene-2-carboxamide (3b)

IR $v\left(\mathrm{~cm}^{-1}\right): 3235(\mathrm{~N}-\mathrm{H}), 1645(\mathrm{C}=\mathrm{O}) ;{ }^{1} \mathrm{H}-\mathrm{NMR} \delta(\mathrm{ppm}): 9.13(\mathrm{~s}, 1 \mathrm{H}$, CONH), 8.10-7.26 (m, 12H, Ar-H), 6.96-6.90 (d, 2H, CH=CH), 3.86 (s, 3H, $\left.\mathrm{OCH}_{3}\right) ;{ }^{13} \mathrm{C}-\mathrm{NMR} \delta$ (ppm): 189.5, 161.6, 160.2, 145.1, 141.6, 135.9, 135.0, $133.3,131.2,129.8,129.7,126.6,122.8,121.3,122.1,120.8,113.5,113.2$, 124.4, 124.3, 55.8; MS: m/z, $447.90\left(\mathrm{M}^{+}\right)$. Anal. calcd. for $\mathrm{C}_{25} \mathrm{H}_{18} \mathrm{ClNO}_{3} \mathrm{~S}: \mathrm{C}$, 67.03; H, 04.05; N, 03.13; S, 07.16; found: C, 67.00; H, 04.01; N, 03.09; S, $07.13 \%$.

4.3.2. 3-Chloro-N-\{4-[(2E)-3-(4-chlorophenyl)prop-2-enoyl]phenyl\}-1benzothiophene-2-carboxamide $(3 c)$

IR $v\left(\mathrm{~cm}^{-1}\right): 3225(\mathrm{~N}-\mathrm{H}), 1640(\mathrm{C}=\mathrm{O}), 1568(\mathrm{C}=\mathrm{C}), 1065(=\mathrm{C}-\mathrm{Cl}), 681$ (C-S-C); ${ }^{1} \mathrm{H}-\mathrm{NMR} \delta(\mathrm{ppm}): 9.06(\mathrm{~s}, 1 \mathrm{H}, \mathrm{CONH}), 8.04-7.18(\mathrm{~m}, 12 \mathrm{H}, \mathrm{Ar}-\mathrm{H})$, 6.66-6.44 (d, $2 \mathrm{H}, \mathrm{CH}=\mathrm{CH}) ;{ }^{13} \mathrm{C}-\mathrm{NMR} \delta(\mathrm{ppm}): 189.3,161.2,145.1,143.3$, $141.6,136.6,135.9,134.2,133.5,133.4,131.4,130.0,129.9,128.5,128.0$, 126.7, 126.6, 126.4, 124.4, 124.3, 122.8, 122.1, 121.3; MS: m/z, $452.35\left(\mathrm{M}^{+}\right)$. Anal. calcd. for $\mathrm{C}_{24} \mathrm{H}_{15} \mathrm{Cl}_{2} \mathrm{NO}_{2} \mathrm{~S}$ : C, 63.72; H, 03.34; N, 03.10; S, 07.09; found: C, 63.67; H, 03.30; N, 03.07; S, 07.03\%.

4.3.3. 3-Chloro- $N$-\{4-[(2E)-3-(4-nitrophenyl)prop-2-enoyl]phenyl\}-1benzothiophene-2-carboxamide (3d)

IR v $\left(\mathrm{cm}^{-1}\right): 3231(\mathrm{~N}-\mathrm{H}), 1655(\mathrm{C}=\mathrm{O}), 1570(\mathrm{C}=\mathrm{C}), 685(\mathrm{C}-\mathrm{S}-\mathrm{C}) ;{ }^{1} \mathrm{H}-\mathrm{NMR}$ $\delta(\mathrm{ppm}): 8.80(\mathrm{~s}, 1 \mathrm{H}, \mathrm{CONH}), 8.36-7.64(\mathrm{~m}, 12 \mathrm{H}, \mathrm{Ar}-\mathrm{H}$ and d, $2 \mathrm{H}, \mathrm{CH}=\mathrm{CH})$; ${ }^{13} \mathrm{C}-\mathrm{NMR} \delta(\mathrm{ppm}): 188.7,161.5,147.8,143.7,141.6,141.5,137.7,135.9$, $134.6,133.5,133.4,131.4,129.9,129.5,126.7,124.4,124.3,123.1,122.8$, 122.7, 122.1, 121.2; MS: m/z, $462.90\left(\mathrm{M}^{+}\right)$. Anal. calcd. for $\mathrm{C}_{24} \mathrm{H}_{15} \mathrm{ClN}_{2} \mathrm{O}_{4} \mathrm{~S}$ : C, 62.27; H, 03.27; N, 06.05; S, 06.93; found: C, 62.24; H, 03.23; N, 06.00; S, $06.89 \%$.

\subsection{General procedure for synthesis of compounds $4 a-d$.}

Exemplary detail for 3-Chloro-N-[4-(2-oxo-6-phenyl-1,2,5,6tetrahydropyrimidin-4-yl)phenyl]-1-benzothiophene-2-carboxamide (4a)

A mixture of compound $3 \mathrm{a}(2.50 \mathrm{~g}, 0.006 \mathrm{~mol})$ in 1,4-dioxane $(10 \mathrm{~mL})$ and urea $(0.36 \mathrm{~g}, 0.006 \mathrm{~mol})$ was refluxed for $15 \mathrm{~h}$. The completion of the reaction was monitored by TLC. The reaction mixture was allowed to cool down to room temperature and, then poured into crushed ice with constant stirring. The reddish yellow solid was obtained, filtered, washed with water, dried and recrystallized from 1,4-dioxane to produce $\mathbf{4 a}$. Compounds $\mathbf{4 b}$-d were prepared in the same manner.

IR $\vee\left(\mathrm{cm}^{-1}\right): 3240(\mathrm{~N}-\mathrm{H}), 1643(\mathrm{C}=\mathrm{O}), 1565(\mathrm{C}=\mathrm{C}), 1065(=\mathrm{C}-\mathrm{Cl})$; ${ }^{1} \mathrm{H}-\mathrm{NMR} \delta$ (ppm): $9.86(\mathrm{~s}, 1 \mathrm{H}, \mathrm{NH}), 9.46(\mathrm{~s}, 1 \mathrm{H}, \mathrm{CONH}), 8.04-7.32(\mathrm{~m}, 13 \mathrm{H}$, $\mathrm{Ar}-\mathrm{H}), 4.10$ (s, $\left.2 \mathrm{H}, \mathrm{CH}_{2}\right) ;{ }^{13} \mathrm{C}-\mathrm{NMR} \delta$ (ppm): 164.1, 161.1, 163, 143.5, 141.6, 140.2 , 136.2, 135.9, 133.4, 129.9, 129.4, 128.5, 128.5, 126.9, 126.7, 126.7, 124.4, 124.3, 122.8, 121.7, 43.6, 42.7; MS: m/z, $459.94\left(\mathrm{M}^{+}\right)$. Anal. calcd. for $\mathrm{C}_{25} \mathrm{H}_{18} \mathrm{ClN}_{3} \mathrm{O}_{2} \mathrm{~S}$ : C, 65.28; H, 03.94; N, 09.14; S, 06.97; found: C, 65.22; H, $03.90 ; \mathrm{N}, 09.09 ; \mathrm{S}, 06.92 \%$. tetrahydropyrimidin-4-yl)phenyl]-1-benzothiophene-2-carboxamide (4b)

IR v $\left(\mathrm{cm}^{-1}\right): 3246(\mathrm{~N}-\mathrm{H}), 1650(\mathrm{C}=\mathrm{O}), 1571(\mathrm{C}=\mathrm{C}), 1045(=\mathrm{C}-\mathrm{Cl}), 685$ (C-S-C); ${ }^{1} \mathrm{H}-\mathrm{NMR} \delta$ (ppm): 10.21 (s, $\left.1 \mathrm{H}, \mathrm{NH}\right), 9.89$ (s, $\left.1 \mathrm{H}, \mathrm{CONH}\right), 7.98-7.14$ $(\mathrm{m}, 12 \mathrm{H}, \mathrm{Ar}-\mathrm{H}), 3.98\left(\mathrm{~s}, 2 \mathrm{H}, \mathrm{CH}_{2}\right), 3.80\left(\mathrm{~s}, 3 \mathrm{H}, \mathrm{OCH}_{3}\right) ;{ }^{13} \mathrm{C}-\mathrm{NMR} \delta(\mathrm{ppm})$ : $164.3,162,161.9,158.6,141.6,140.2,136.2,135.9,135.8,133.4,129.9$, 129.4, 126.7, 126.6, 124.4, 124.3, 122.8, 121.7, 114.1, 55.8, 43.2, 42.2; MS: $\mathrm{m} / \mathrm{z}, 489.97\left(\mathrm{M}^{+}\right)$. Anal. calcd. for $\mathrm{C}_{26} \mathrm{H}_{20} \mathrm{ClN}_{3} \mathrm{O}_{3} \mathrm{~S}: \mathrm{C}, 63.73 ; \mathrm{H}, 04.11 ; \mathrm{N}, 08.58$; $\mathrm{S}, 06.54$; found: $\mathrm{C}, 63.70 ; \mathrm{H}, 04.09 ; \mathrm{N}, 08.55 ; \mathrm{S}, 06.50 \%$.

4.4.2. 3-Chloro-N-[4-(2-oxo-4-chlorophenyl-1,2,5,6-tetrahydropyrimidin4-yl)phenyl]-1-benzothiophene-2-carboxamide (4c)

IR $v\left(\mathrm{~cm}^{-1}\right): 3235(\mathrm{~N}-\mathrm{H}), 1665(\mathrm{C}=\mathrm{O}) ;{ }^{1} \mathrm{H}-\mathrm{NMR} \delta(\mathrm{ppm}): 10.35(\mathrm{~s}, 1 \mathrm{H}$, $\mathrm{NH}), 10.03$ (s, 1H, CONH), 8.12-6.85 (m, 12H, Ar-H), $4.25\left(\mathrm{~s}, 2 \mathrm{H}, \mathrm{CH}_{2}\right)$; ${ }^{13} \mathrm{C}-\mathrm{NMR} \delta(\mathrm{ppm}): 164.7,163.3,161.5,141.6,140.2,136.2,135.9,133.4$, 132.3, 129.9, 129.4, 128.6, 127.2, 126.7, 124.4, 124.3, 122.8, 121.7, 43.3, 42.1; MS: $\mathrm{m} / \mathrm{z}, 494.39\left(\mathrm{M}^{+}\right)$. Anal. calcd. for $\mathrm{C}_{25} \mathrm{H}_{17} \mathrm{Cl}_{2} \mathrm{~N}_{3} \mathrm{O}_{2} \mathrm{~S}: \mathrm{C}, 60.73 ; \mathrm{H}, 03.47 ; \mathrm{N}$, 08.50; S, 06.49; found: C, 60.70; H, 03.43; N, 08.43; S, 06.45\%.

4.4.3. 3-Chloro-N-[4-(2-oxo-4-nitroyphenyl-1,2,5,6-tetrahydropyrimidin4-yl)phenyl]-1-benzothiophene-2-carboxamide (4d)

IR $v\left(\mathrm{~cm}^{-1}\right): 3242(\mathrm{~N}-\mathrm{H}), 1650(\mathrm{C}=\mathrm{O}) ;{ }^{1} \mathrm{H}-\mathrm{NMR} \delta(\mathrm{ppm}): 10.09(\mathrm{~s}, 1 \mathrm{H}$, $\mathrm{NH}), 10.48$ (s, 1H, CONH), 8.27-7.44 (m, 12H, Ar-H), $4.22\left(\mathrm{~s}, 2 \mathrm{H}, \mathrm{CH}_{2}\right)$; ${ }^{13} \mathrm{C}-\mathrm{NMR} \delta(\mathrm{ppm}): 164.3,163.6,161.5,149.6,145.9,141.6,140.2,135.9$, $133.4,136.2$, 129.9, 129.4, 126.7, 124.4, 124.3, 123.4, 123.7, 122.8, 121.7, 43.0, 42.1; MS: $\mathrm{m} / \mathrm{z}, 504.94\left(\mathrm{M}^{+}\right)$. Anal. calcd. for $\mathrm{C}_{25} \mathrm{H}_{17} \mathrm{ClN}_{4} \mathrm{O}_{4} \mathrm{~S}: \mathrm{C}, 59.47$ H, 03.39; N, 11.10; S, 06.35; found: C, 59.41; H, 03.32; N, 11.05; S, 06.30\%.

\subsection{General procedure for synthesis of compounds 5a-d.}

Exemplary detail for 3-Chloro-N-[4-(5-phenyl-4,5-dihydroisoxazol-3-yl) phenyl-1-benzothiophene-2-carboxamide (5a)

A mixture of compound 3a $(2.50 \mathrm{~g}, 0.006 \mathrm{~mol})$ in DMF $(25 \mathrm{~mL})$ and hydroxylamine hydrochloride $(0.41 \mathrm{~g}, 0.006 \mathrm{~mol})$ was refluxed for $15 \mathrm{~h}$. The completion of the reaction was monitored by TLC. The reaction mixture was allowed to cool down to room temperature, and then poured into ice cooled water with constant stirring. The precipitate was filtered, washed with water, dried and recrystallized from 1,4-dioxane. The resulting solid was further purified by silica column, using a gradient mixture of chloroform/acetone (80:20) as an eluent to obtain $\mathbf{5 a}$. Compounds $\mathbf{5}$ b-d were prepared in the same manner.

IR $v\left(\mathrm{~cm}^{-1}\right): 3275(\mathrm{~N}-\mathrm{H}), 1650(\mathrm{C}=\mathrm{O}), 1620(\mathrm{C}=\mathrm{N}) ;{ }^{1} \mathrm{H}-\mathrm{NMR} \delta(\mathrm{ppm})$ : 10.45 (s, 1H, CONH), 8.15-7.35 (m, 12H, Ar-H), 2.70 (s, $\left.2 \mathrm{H}, \mathrm{CH}_{2}\right) ;{ }^{13} \mathrm{C}-\mathrm{NMR}$ $\delta(\mathrm{ppm}): 161.7,156.1,142.2,141.6,140.2,135.9,133.4,129.9,129.4,128.9$, 127.6, 127.1, 127.1, 126.7, 126.0, 124.4, 124.3, 122.8, 121.7, 82.3, 42.3; MS: $\mathrm{m} / \mathrm{z}, 432.92\left(\mathrm{M}^{+}\right)$. Anal. calcd. for $\mathrm{C}_{24} \mathrm{H}_{17} \mathrm{ClN}_{2} \mathrm{O} 2 \mathrm{~S}: \mathrm{C}, 66.58 ; \mathrm{H}, 03.96 ; \mathrm{N}$, 06.47 ; S, 07.41; found: C, 66.55; H, 03.96; N, 06.45; S, 07.40\%.

4.5.1. 3-Chloro- $N$-\{4-[5-(4-methoxyphenyl)4,5-dihydroisoxazol-3-yl] phenyl\}-1-benzothi ophene-2-carboxamide (5b)

IR $v\left(\mathrm{~cm}^{-1}\right): 3265(\mathrm{~N}-\mathrm{H}), 1645(\mathrm{C}=\mathrm{O}), 1622(\mathrm{C}=\mathrm{N}) ;{ }^{1} \mathrm{H}-\mathrm{NMR} \delta(\mathrm{ppm})$ : 9.99 (s, 1H, CONH), 8.12-7.45 (m, 12H, Ar-H), $3.70\left(\mathrm{~s}, 3 \mathrm{H}, \mathrm{OCH}_{3}\right), 3.60$ (s, $\left.2 \mathrm{H}, \mathrm{CH}_{2}\right) ;{ }^{13} \mathrm{C}-\mathrm{NMR} \delta(\mathrm{ppm}): 161.0,159.5,156.2,141.6,140.2,135.9,134.7$, $133.4,129.9,129.4,127.0,126.7,126.0,124.4,124.3,122.8,121.7,114.5$, 114.5, 82.2, 55.4, 42.5; MS: m/z, $462.94\left(\mathrm{M}^{+}\right)$. Anal. calcd. for $\mathrm{C}_{25} \mathrm{H}_{19} \mathrm{ClN}_{2} \mathrm{O}_{3} \mathrm{~S}$ : C, 64.86; H, 04.14; N, 06.05; S, 06.93; found: C, 64.85; H, 04.12; N, 06.01; S, $06.90 \%$.

4.5.2. 3-Chloro- $N$-\{4-[5-(4-chlorophenyl)-4,5-dihydroisoxazol-3-yl] phenyl\}-1-benzothio phene-2-carboxamide (5c)

IR $v\left(\mathrm{~cm}^{-1}\right): 3270(\mathrm{~N}-\mathrm{H}), 1640(\mathrm{C}=\mathrm{O}), 1615(\mathrm{C}=\mathrm{N}) ;{ }^{1} \mathrm{H}-\mathrm{NMR} \delta(\mathrm{ppm})$ : 10.07 (s, 1H, CONH), 8.18-7.02 (m, 12H, Ar-H), $3.81\left(\mathrm{~s}, 2 \mathrm{H}, \mathrm{CH}_{2}\right) ;{ }^{13} \mathrm{C}-\mathrm{NMR}$ $\delta(\mathrm{ppm}): 161.7,156.3,141.3,140.2,140.2,135.9,133.4,133.5,129.9,129.4$, 129.4, 129.0, 126.9, 126.7, 126.0, 124.4, 124.3, 122.1, 121.2, 82.3, 42.6; MS: $\mathrm{m} / \mathrm{z}, 467.36\left(\mathrm{M}^{+}\right)$. Anal. calcd. for $\mathrm{C}_{24} \mathrm{H}_{16} \mathrm{Cl}_{2} \mathrm{~N}_{2} \mathrm{O}_{2} \mathrm{~S}: \mathrm{C}, 61.68 ; \mathrm{H}, 03.45 ; \mathrm{N}$, 05.99; S, 06.86; found: C, 61.66; H, 03.40; N, 05.94; S, 06.82\%.

4.5.3. 3-Chloro-N-\{4-nitrophenyl-)-4,5-dihydroisoxazol-3-yl]phenyl\}-1- 
benzothiophene-2-carboxamide (5d)

IR $v\left(\mathrm{~cm}^{-1}\right): 3260(\mathrm{~N}-\mathrm{H}), 1642(\mathrm{C}=\mathrm{O}), 1625(\mathrm{C}=\mathrm{N}) ;{ }^{1} \mathrm{H}-\mathrm{NMR} \delta(\mathrm{ppm})$ : 9.89 (s, $1 \mathrm{H}, \mathrm{CONH}), 7.95-7.02(\mathrm{~m}, 12 \mathrm{H}, \mathrm{Ar}-\mathrm{H}), 3.75\left(\mathrm{~s}, 2 \mathrm{H}, \mathrm{CH}_{2}\right) ;{ }^{13} \mathrm{C}-\mathrm{NMR}$ $\delta(\mathrm{ppm}): 161.5,156.1,148.5,146.8,141.6,140.2,135.9,133.4,129.9,129.4$, 128.0, 126.7, 126.0, 124.4, 124.3, 124.1, 122.8, 121.7, 121.1, 82.3, 42.2; MS: $\mathrm{m} / \mathrm{z}, 477.91\left(\mathrm{M}^{+}\right)$. Anal. calcd. for $\mathrm{C}_{24} \mathrm{H}_{16} \mathrm{ClN}_{3} \mathrm{O}_{4} \mathrm{~S}: \mathrm{C}, 60.31 ; \mathrm{H}, 03.37 ; \mathrm{N}$, 08.79; S, 06.71; found: C, $60.25 ; \mathrm{H}, 03.34 ; \mathrm{N}, 08.75 ; \mathrm{S}, 06.68 \%$.

\subsection{General procedure for synthesis of compounds $6 a-h$.}

Exemplary detail for 3-chloro-N-[4-(1,5-diphenyl-4,5-dihydro-1Hpyrazol-3-yl)phenyll-1-benzothiophene-2-carboxamide (6a)

A mixture of compound 3a $(2.50 \mathrm{~g}, 0.006 \mathrm{~mol})$ in DMF $(25 \mathrm{~mL})$ and phenylhydrazine $(0.64 \mathrm{~g}, 0.006 \mathrm{~mol})$ was refluxed for $15 \mathrm{~h}$. The completion of the reaction was monitored by TLC. The reaction mixture was allowed to cool down to room temperature and poured into ice cooled water with constant stirring. The resulting precipitate was filtered, washed with water, dried and recrystallized from 1,4-dioxane to produce $\mathbf{6 a}$. Compounds $\mathbf{6} \mathbf{b}-\mathbf{h}$ were prepared in the same manner.

IR $v\left(\mathrm{~cm}^{-1}\right): 3277(\mathrm{~N}-\mathrm{H}), 1643(\mathrm{C}=\mathrm{O}), 1613(\mathrm{C}=\mathrm{N}) ;{ }^{1} \mathrm{H}-\mathrm{NMR} \delta(\mathrm{ppm})$ : $10.65(\mathrm{~s}, 1 \mathrm{H}, \mathrm{CONH}), 8.18-7.27(\mathrm{~m}, 18 \mathrm{H}, \mathrm{Ar}-\mathrm{H}), 2.40\left(\mathrm{~s}, 2 \mathrm{H}, \mathrm{CH}_{2}\right) ;{ }^{13} \mathrm{C}-\mathrm{NMR}$ $\delta(\mathrm{ppm}): 161.6,151.2,143.8,141.6,140.2,143.5,135.9,133.4,132.0,129.9$, $129.5,129.5,129.4,128.5,128.5,126.9,126.8,126.7,124.4,124.3,122.8$, 121.7, 120.8, 116.7, 53.2, 40.5; MS: $\mathrm{m} / \mathrm{z}, 508.03\left(\mathrm{M}^{+}\right)$. Anal. calcd. for $\mathrm{C}_{30} \mathrm{H}_{22} \mathrm{ClN}_{3} \mathrm{OS}: \mathrm{C}, 70.92 ; \mathrm{H}, 04.36 ; \mathrm{N}, 08.27 ; \mathrm{S}, 06.31$; found: $\mathrm{C}, 70.89 ; \mathrm{H}$, $04.31 ; \mathrm{N}, 08.23 ; \mathrm{S}, 06.27 \%$.

4.6.1. 3-Chloro- $N$-[4-(1-phenyl-4-methoxyphenyl-4,5-dihydro- $1 \mathrm{H}$ pyrazol-3-yl)phenyl]-1-benzothiophene-2-carboxamide (6b)

IR $v\left(\mathrm{~cm}^{-1}\right): 3275(\mathrm{~N}-\mathrm{H}), 1635(\mathrm{C}=\mathrm{O}), 1615(\mathrm{C}=\mathrm{N}) ;{ }^{1} \mathrm{H}-\mathrm{NMR} \delta(\mathrm{ppm})$ : 10.38 (s, 1H, CONH), 7.95-7.02 (m, 17H, Ar-H), 3.80 (s, 3H, $\left.\mathrm{OCH}_{3}\right), 2.80$ (s, $\left.2 \mathrm{H}, \mathrm{CH}_{2}\right) ;{ }^{13} \mathrm{C}-\mathrm{NMR} \delta(\mathrm{ppm}): 161.7,158.3,151.1,143.1,141.1,140.1,135.1$, 133.1, 132.0, 129.9, 129.5, 129.4, 126.7, 126.6, 124.1, 124.1, 122.2, 121.3, 121.4, 120.8, 116.7, 114.1, 55.8, 53.2, 40.5; MS: m/z, 538.05 (M+); Anal calcd. for $\mathrm{C}_{31} \mathrm{H}_{24} \mathrm{ClN}_{3} \mathrm{O}_{2} \mathrm{~S}: \mathrm{C}, 69.20 ; \mathrm{H}, 04.50 ; \mathrm{N}, 07.81 ; \mathrm{S}, 05.96$. Found: $\mathrm{C}$, $69.15 ; \mathrm{H}, 04.44 ; \mathrm{N}, 07.78 ; \mathrm{S}, 05.90 \%$.

4.6.2. 3-Chloro-N-[4-(1-phenyl-4-chlorophenyl-4,5-dihydro-1H-pyrazol3-yl)phenyl]-1-benzothiophene-2-carboxamide (6c)

IR $v\left(\mathrm{~cm}^{-1}\right): 3265(\mathrm{~N}-\mathrm{H}), 1640(\mathrm{C}=\mathrm{O}), 1625(\mathrm{C}=\mathrm{N}) ;{ }^{1} \mathrm{H}-\mathrm{NMR} \delta(\mathrm{ppm}):$ $10.22(\mathrm{~s}, 1 \mathrm{H}, \mathrm{CONH}), 8.02-7.13(\mathrm{~m}, 17 \mathrm{H}, \mathrm{Ar}-\mathrm{H}), 2.69\left(\mathrm{~s}, 2 \mathrm{H}, \mathrm{CH}_{2}\right) ;{ }^{13} \mathrm{C}-\mathrm{NMR}$ $\delta(\mathrm{ppm}): 161.4,151.2,143.2,141.2,141.2,140.2,135.9,133.4,132.3,132.0$, $129.9,129.5,129.4,128.6,128.6,127.2,126.7,124.4,124.3,122.8,121.7$, $120.8,116.7,116.7,53.4,40.3$; MS: $\mathrm{m} / \mathrm{z}, 542.46\left(\mathrm{M}^{+}\right)$. Anal. calcd. for $\mathrm{C}_{30} \mathrm{H}_{21} \mathrm{Cl}_{2} \mathrm{~N}_{3} \mathrm{OS}$ : C, 66.42; H, 03.90; N, 07.75; S, 05.91; found: $\mathrm{C}, 66.38 ; \mathrm{H}$, $03.89 ; \mathrm{N}, 7.71 ; \mathrm{S}, 05.89 \%$.

4.6.3. 3-Chloro-N-[4-(1-phenyl-4-nitrophenyl-4,5-dihydro-1H-pyrazol-3yl)phenyl]-1-benzothiophene-2-carboxamide (6d)

IR $v\left(\mathrm{~cm}^{-1}\right): 3264(\mathrm{~N}-\mathrm{H}), 1659(\mathrm{C}=\mathrm{O}), 1614(\mathrm{C}=\mathrm{N}) ;{ }^{1} \mathrm{H}-\mathrm{NMR} \delta(\mathrm{ppm}):$ $10.32(\mathrm{~s}, 1 \mathrm{H}, \mathrm{CONH}), 8.14-7.87(\mathrm{~m}, 17 \mathrm{H}, \mathrm{Ar}-\mathrm{H}), 2.71\left(\mathrm{~s}, 2 \mathrm{H}, \mathrm{CH}_{2}\right) ;{ }^{13} \mathrm{C}-\mathrm{NMR}$ $\delta$ (ppm): $161.1,151.2,149.3,145.1,143.8,141.6,140.2,135.9,133.4,132.0$, $129.9,129.5,129.4,126.7,124.4,124.3,123.7,123.7,123.4,123.4,122.8$, 121.7, 120.8, 116.7, 116.4, 53.1, 40.2; MS: $\mathrm{m} / \mathrm{z}, 553.01\left(\mathrm{M}^{+}\right)$. Anal. calcd. for $\mathrm{C}_{30} \mathrm{H}_{21} \mathrm{ClN}_{4} \mathrm{O}_{3} \mathrm{~S}: \mathrm{C}, 65.15 ; \mathrm{H}, 03.83 ; \mathrm{N}, 10.13 ; \mathrm{S}, 05.80$; found: $\mathrm{C}, 65.10 ; \mathrm{H}$, $03.80 ; \mathrm{N}, 10.10 ; \mathrm{S}, 05.76 \%$.

4.6.4. 3-Chloro- $N$-\{4-[1-(4-fluorophenyl)-4-phenyl-4,5-dihydro- $1 \mathrm{H}$ pyrazol-3-yl]phenyl\}-1-benzothiophene-2-carboxamide (6e)

IR $v\left(\mathrm{~cm}^{-1}\right): 3250(\mathrm{~N}-\mathrm{H}), 1642(\mathrm{C}=\mathrm{O}), 1595(\mathrm{C}=\mathrm{N}) ;{ }^{1} \mathrm{H}-\mathrm{NMR} \delta(\mathrm{ppm}):$ $10.40(\mathrm{~s}, 1 \mathrm{H}, \mathrm{CONH}), 8.39-7.45(\mathrm{~m}, 17 \mathrm{H}, \mathrm{Ar}-\mathrm{H}), 2.50\left(\mathrm{~s}, 2 \mathrm{H}, \mathrm{CH}_{2}\right) ;{ }^{13} \mathrm{C}-\mathrm{NMR}$ $\delta$ (ppm): 161.9, 155.2, 151.2, 143.1, 141.3, 140.2, 139.4, 135.9, 133.4, 132.0, $129.9,129.4,128.5,126.9,126.9,126.7,124.4,124.3,122.4,121.3,116.2$ 115.1, 53.3, 40.8; MS: $\mathrm{m} / \mathrm{z}, 526.02\left(\mathrm{M}^{+}\right)$. Anal. calcd. for $\mathrm{C}_{30} \mathrm{H}_{21} \mathrm{ClFN}_{3} \mathrm{OS}: \mathrm{C}$, 68.50; H, 04.02; N, 07.99; S, 06.10; found: C, 68.48; H, 04.00; N, 07.95; S, $06.05 \%$.
4.6.5. 3-Chloro-N-\{4-[1-(4-fluorophenyl)-4-methoxyphenyl-4,5-dihydro1H-pyrazol-3-yl] phenyl\}-1-benzothiophene-2-carboxamide (6f)

IR $v\left(\mathrm{~cm}^{-1}\right): 3275(\mathrm{~N}-\mathrm{H}), 1650(\mathrm{C}=\mathrm{O}), 1623(\mathrm{C}=\mathrm{N}) ;{ }^{1} \mathrm{H}-\mathrm{NMR} \delta(\mathrm{ppm}):$ 10.45 (s, $1 \mathrm{H}, \mathrm{CONH}), 8.03-7.29(\mathrm{~m}, 16 \mathrm{H}, \mathrm{Ar}-\mathrm{H}), 3.75\left(\mathrm{~s}, 3 \mathrm{H}, \mathrm{OCH}_{3}\right), 2.52(\mathrm{~s}$, $\left.2 \mathrm{H}, \mathrm{CH}_{2}\right) ;{ }^{13} \mathrm{C}-\mathrm{NMR} \delta$ (ppm): $161.2,158.2,155.1,151.2,141.4,140.2,139.4$, $135.9,135.8,133.4,132.0,129.9,129.4,126.7,126.6,124.4,124.3,122.8$, 121.5, 116.2, 115.1, 114.3, 55.2, 40.6; MS: $\mathrm{m} / \mathrm{z}, 556.04\left(\mathrm{M}^{+}\right)$. Anal. calcd. for $\mathrm{C}_{31} \mathrm{H}_{23} \mathrm{ClFN}_{3} \mathrm{O}_{2} \mathrm{~S}: \mathrm{C}, 66.96 ; \mathrm{H}, 04.17$; N, 07.56; S, 05.77; found: $\mathrm{C}, 66.91 ; \mathrm{H}$, $04.12 ; \mathrm{N}, 07.51 ; \mathrm{S}, 05.71 \%$.

4.6.6. 3-Chloro-N-\{4-[1-(4-fluorophenyl)-4-chlorophenyl-4,5-dihydro1H-pyrazol-3-yl]phenyl\}-1-benzothiophene-2-carboxamide (6g)

IR $v\left(\mathrm{~cm}^{-1}\right): 3272(\mathrm{~N}-\mathrm{H}), 1645(\mathrm{C}=\mathrm{O}), 1624(\mathrm{C}=\mathrm{N}) ;{ }^{1} \mathrm{H}-\mathrm{NMR} \delta(\mathrm{ppm})$ : 10.35 (s, 1H, CONH), 7.98-7.03 (m, 16H, Ar-H), 2.45 (s, $\left.2 \mathrm{H}, \mathrm{CH}_{2}\right) ;{ }^{13} \mathrm{C}-\mathrm{NMR}$ $\delta(\mathrm{ppm}): 161.7,155.4,151.5,141.3,141.5,140.2,139.4,135.9,133.4,132.3$, $132.0,129.9,129.4,128.6,127.2,126.7,124.4,124.3,122.8,121.7,121.7$, 116.3, 116.3, 115.1, 53.2, 40.4; MS: $\mathrm{m} / \mathrm{z}, 560.47\left(\mathrm{M}^{+}\right)$. Anal. calcd. for $\mathrm{C}_{30} \mathrm{H}_{20} \mathrm{Cl}_{2} \mathrm{FN}_{3} \mathrm{OS}: \mathrm{C}, 64.29 ; \mathrm{H}, 03.60 ; \mathrm{N}, 07.50 ; \mathrm{S}, 05.72$; found: $\mathrm{C}, 48.08 ; \mathrm{H}$, $03.50 ; \mathrm{N}, 07.00 ; \mathrm{S}, 5.70 \%$

4.6.7. 3-Chloro-N-\{4-[1-(4-fluorophenyl)-4-nitrophenyl-4,5-dihydro-1Hpyrazol-3-yl] phenyl\}-1-benzothiophene-2-carboxamide (6h)

IR $v\left(\mathrm{~cm}^{-1}\right): 3281(\mathrm{~N}-\mathrm{H}), 1656(\mathrm{C}=\mathrm{O}), 1610(\mathrm{C}=\mathrm{N}) ;{ }^{1} \mathrm{H}-\mathrm{NMR} \delta(\mathrm{ppm})$ : 11.43 (s, $1 \mathrm{H}, \mathrm{CONH}), 8.11-6.99(\mathrm{~m}, 16 \mathrm{H}, \mathrm{Ar}-\mathrm{H}), 2.43$ (s, $\left.2 \mathrm{H}, \mathrm{CH}_{2}\right) ;{ }^{13} \mathrm{C}-\mathrm{NMR}$ $\delta$ (ppm): 161.2, 155.1, 151.4, 149.6, 145.9, 141.6, 140.2, 139.4, 135.9, 133.4, $132.0,129.9,129.4,126.7,124.4,124.3,123.7,123.4,122.8,121.7,121.7$, 116.3, 115.3, 115.1, 53.7, 40.5; MS: $\mathrm{m} / \mathrm{z}, 571.02\left(\mathrm{M}^{+}\right)$. Anal. calcd. for $\mathrm{C}_{30} \mathrm{H}_{20} \mathrm{ClFN}_{4} \mathrm{O}_{3} \mathrm{~S}: \mathrm{C}, 63.10 ; \mathrm{H}, 03.53 ; \mathrm{N}, 09.81 ; \mathrm{S}, 05.62$; found: $\mathrm{C}, 63.07 ; \mathrm{H}$, $03.50 ; \mathrm{N}, 09.78 ; \mathrm{S}, 05.60 \%$.

\subsection{General procedure for synthesis of compounds $7 a-d$.}

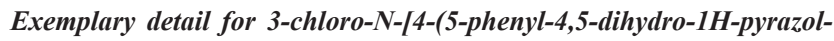
3-yl)phenyl]-1-benzothiophene-2-carboxamide (7a)

A mixture of compound 3a $(2.50 \mathrm{~g}, 0.006 \mathrm{~mol})$ in 1.4-dioxane $(20 \mathrm{~mL})$ and hydrazine hydrate $(0.29 \mathrm{~g}, 0.006 \mathrm{~mol})$ was refluxed for $10 \mathrm{~h}$. After completion of reaction, the reaction mixture was cooled, poured into crushed ice and then neutralized with $\mathrm{HCl}$. The precipitate was filtered, washed with water, dried and recrystallized from methanol. It was further purified by column chromatography using a gradient mixture of petroleum ether/ethyl acetate $(80: 20)$ as an eluent to give compound $7 \mathbf{a}$. Compounds $7 \mathbf{b}$-d were prepared in the same manner.

IR $v\left(\mathrm{~cm}^{-1}\right): 3240(\mathrm{~N}-\mathrm{H}), 1650(\mathrm{C}=\mathrm{O}), 1626(\mathrm{C}=\mathrm{N}) ;{ }^{1} \mathrm{H}-\mathrm{NMR} \delta(\mathrm{ppm})$ : $10.84(\mathrm{~s}, 1 \mathrm{H}, \mathrm{NH}), 9.70(\mathrm{~s}, 1 \mathrm{H}, \mathrm{CONH}), 8.23-7.01(\mathrm{~m}, 13 \mathrm{H}, \mathrm{Ar}-\mathrm{H}), 3.45(\mathrm{~s}$, $\left.2 \mathrm{H}, \mathrm{CH}_{2}\right) ;{ }^{13} \mathrm{C}-\mathrm{NMR} \delta(\mathrm{ppm}): 161.0,151.1,143.4,141.3,140.1,143.5,135.9$, $133.4,132.0,129.9,129.4,129.5,129.5,128.5,128.5,126.9,126.7,124.4$,

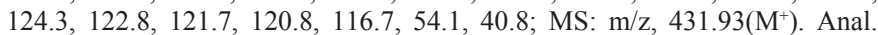
calcd. for $\mathrm{C}_{24} \mathrm{H}_{18} \mathrm{ClN}_{3} \mathrm{OS}$ : C, 67.74; $\mathrm{H}, 04.20 ; \mathrm{N}, 09.73 ; \mathrm{S}, 07.4$; found: $\mathrm{C}$, $67.70 ; \mathrm{H}, 04.17 ; \mathrm{N}, 09.70 ; \mathrm{S}, 07.39 \%$.

4.7.1. 3-Chloro-N-[4-(4-methoxyphenyl-4,5-dihydro-1H-pyrazol-3-yl) phenyl]-1-benzoth iophene-2-carboxamide (7b)

IR $v\left(\mathrm{~cm}^{-1}\right): 3245(\mathrm{~N}-\mathrm{H}), 1660(\mathrm{C}=\mathrm{O}), 1616(\mathrm{C}=\mathrm{N}) ;{ }^{1} \mathrm{H}-\mathrm{NMR} \delta(\mathrm{ppm})$ : $10.49(\mathrm{~s}, 1 \mathrm{H}, \mathrm{NH}), 9.62(\mathrm{~s}, 1 \mathrm{H}, \mathrm{CONH}), 8.93-7.60(\mathrm{~m}, 12 \mathrm{H}, \mathrm{Ar}-\mathrm{H}), 3.91$ $\left(\mathrm{s}, 2 \mathrm{H}, \mathrm{OCH}_{3}\right), 3.57\left(\mathrm{~s}, 2 \mathrm{H}, \mathrm{CH}_{2}\right) ;{ }^{13} \mathrm{C}-\mathrm{NMR} \delta(\mathrm{ppm}): 161.6,158.3,151.3$, $141.5,140.2,135.9,135.8,133.4,132.0,129.9,129.4,126.7,126.6,124.4$, 124.3, 121.7, 114.1, 55.8, 49.9, 42.1; MS: $\mathrm{m} / \mathrm{z}, 461.96\left(\mathrm{M}^{+}\right)$. Anal. calcd. for $\mathrm{C}_{25} \mathrm{H}_{20} \mathrm{ClN}_{3} \mathrm{O}_{2} \mathrm{~S}: \mathrm{C}, 65.00 ; \mathrm{H}, 04.36 ; \mathrm{N}, 09.10 ; \mathrm{S}, 06.94$; found: $\mathrm{C}, 64.98 ; \mathrm{H}$, $04.30 ; \mathrm{N}, 09.03 ; \mathrm{S}, 06.89 \%$.

4.7.2. 3-Chloro-N-[4-(4-chlorophenyl-4,5-dihydro-1H-pyrazol-3-yl) phenyl]-1-benzo thiophene-2-carboxamide (7c)

IR $v\left(\mathrm{~cm}^{-1}\right): 3265(\mathrm{~N}-\mathrm{H}), 1667(\mathrm{C}=\mathrm{O}), 1622(\mathrm{C}=\mathrm{N}) ;{ }^{1} \mathrm{H}-\mathrm{NMR} \delta(\mathrm{ppm})$ : 10.75 (s, $1 \mathrm{H}, \mathrm{NH}), 10.11(\mathrm{~s}, 1 \mathrm{H}, \mathrm{CONH}), 8.20-7.33(\mathrm{~m}, 12 \mathrm{H}, \mathrm{Ar}-\mathrm{H}), 2.85(\mathrm{~s}$, $\left.2 \mathrm{H}, \mathrm{CH}_{2}\right) ;{ }^{13} \mathrm{C}-\mathrm{NMR} \delta(\mathrm{ppm}): 161.6,151.3,141.2,140.2,135.4,133.2,132.3$, 132.0, 129.9, 129.4, 128.6, 127.2, 126.7, 124.4, 124.3, 122.8, 121.7, 49.7, 42.5; MS: m/z, $466.37\left(\mathrm{M}^{+}\right)$. Anal. calcd. for $\mathrm{C}_{24} \mathrm{H}_{17} \mathrm{Cl}_{2} \mathrm{~N}_{3} \mathrm{OS}: \mathrm{C}, 61.81 ; \mathrm{H}, 03.67 ; \mathrm{N}$, 
09.01; S, 06.88; found: C, 61.78; H, 03.61; N, 09.00; S, 06.85\%.

4.7.3. 3-Chloro-N-[4-(4-nitrophenyl-4,5-dihydro-1H-pyrazol-3-yl) phenyl]-1-benzothio phene-2-carboxamide (7d)

IR $v\left(\mathrm{~cm}^{-1}\right): 3270(\mathrm{~N}-\mathrm{H}), 1660(\mathrm{C}=\mathrm{O}), 1628(\mathrm{C}=\mathrm{N}) ;{ }^{1} \mathrm{H}-\mathrm{NMR} \delta(\mathrm{ppm})$ : $10.66(\mathrm{~s}, 1 \mathrm{H}, \mathrm{NH}), 9.88(\mathrm{~s}, 1 \mathrm{H}, \mathrm{CONH}), 8.45-7.07$ (m, 12H, Ar-H), 2.88 (s, $\left.2 \mathrm{H}, \mathrm{CH}_{2}\right) ;{ }^{13} \mathrm{C}-\mathrm{NMR} \delta(\mathrm{ppm}): 161.7,151.5,149.5,145.5,141.5,140.5,135.4$, 133.4, 132.0, 129.9, 129.4, 126.7, 124.4, 124.3, 123.7, 123.4, 122.8, 121.7, 49.1, 41.8; MS: $\mathrm{m} / \mathrm{z}, 476.93\left(\mathrm{M}^{+}\right)$. Anal. calcd. for $\mathrm{C}_{24} \mathrm{H}_{17} \mathrm{ClN}_{4} \mathrm{O}_{3} \mathrm{~S}: \mathrm{C}, 60.44$; H, 03.59; N, 11.75; S, 06.72; found: C, 60.40; H, 03.53; N, 11.70; S, 06.68\%.

\subsection{General procedure for synthesis of compounds 8a-d.}

Exemplary detail for 3-chloro- $N$-[4-(6-phenyl-2-thioxo-1,2,5,6tetrahydropyrimidin-4-yl)phenyl]-1-benzothiophene-2-carboxamide (8a)

A mixture of compound 3a (2.92 g, $0.007 \mathrm{~mol})$ in 1,4-dioxane $(20 \mathrm{~mL})$ and thiourea $(0.53 \mathrm{~g}, 0.007 \mathrm{~mol})$ was refluxed for $13 \mathrm{~h}$. The completion of the reaction was monitored by TLC technique. The reaction mixture was allowed to cool down to room temperature, and then poured into ice cooled water with stirring. The light yellow solid was filtered, washed with water, dried and recrystallized from 1,4-dioxane which was purified by column chromatography on silica gel, using a gradient mixture of petroleum ether/ethyl acetate $(85: 15)$ as an eluent to give compound $\mathbf{8 a}$. Compounds $\mathbf{8 b}$-d were prepared in the same manner.

IR $v\left(\mathrm{~cm}^{-1}\right): 3265(\mathrm{~N}-\mathrm{H}), 1660(\mathrm{C}=\mathrm{O}), 1445(\mathrm{C}=\mathrm{S}) ;{ }^{1} \mathrm{H}-\mathrm{NMR} \delta(\mathrm{ppm}): 9.67$ $(\mathrm{s}, 1 \mathrm{H}, \mathrm{NH}), 8.63(\mathrm{~s}, 1 \mathrm{H}, \mathrm{CONH}), 7.98-7.37(\mathrm{~m}, 13 \mathrm{H}, \mathrm{Ar}-\mathrm{H}), 4.20\left(\mathrm{~s}, 2 \mathrm{H}, \mathrm{CH}_{2}\right)$; ${ }^{13} \mathrm{C}-\mathrm{NMR} \delta$ (ppm): $161.9,188,164.6,143.5,141.6,140.2,136.2,135.9,133.4$, 129.9, 129.4, 128.5, 126.9, 126.9, 126.7, 124.4, 124.3, 122.8, 121.7, 54.2, 41.6; MS: $\mathrm{m} / \mathrm{z}, 476.01\left(\mathrm{M}^{+}\right)$. Anal. calcd. for $\mathrm{C}_{25} \mathrm{H}_{18} \mathrm{ClN}_{3} \mathrm{OS}_{2}: \mathrm{C}, 63.08 ; \mathrm{H}, 03.81 ; \mathrm{N}$, 08.83; S, 13.47; found: C, 63.01; H, 03.78; N, 08.80; S, $13.42 \%$.

4.7.1. 3-Chloro- $N$-\{4-[6-(4-methoxyphenyl)-2-thioxo-1,2,5,6tetrahydropyrimidin-4-yl] phenyl\}-1-benzothiophene-2-carboxamide (8b)

IR $v\left(\mathrm{~cm}^{-1}\right): 3260(\mathrm{~N}-\mathrm{H}), 1655(\mathrm{C}=\mathrm{O}), 1440(\mathrm{C}=\mathrm{S}) ;{ }^{1} \mathrm{H}-\mathrm{NMR} \delta(\mathrm{ppm})$ : $10.02(\mathrm{~s}, 1 \mathrm{H}, \mathrm{NH}), 9.12(\mathrm{~s}, 1 \mathrm{H}, \mathrm{CONH}), 8.01-7.56(\mathrm{~m}, 12 \mathrm{H}, \mathrm{Ar}-\mathrm{H}), 4.13(\mathrm{~s}, 2 \mathrm{H}$, $\mathrm{CH}_{2}$ ), 3.60 (s. $\left.3 \mathrm{H}, \mathrm{OCH}_{3}\right) ;{ }^{13} \mathrm{C}-\mathrm{NMR} \delta$ (ppm): 186, 166.6, 161.2, 158.6, 141.6 , $140.2,136.2,135.9,135.8,133.4,129.9,129.4,126.7,126.6,124.4,124.3$, 122.8, 121.7, 114.1, 55.2, 52.9, 42.1; MS: $\mathrm{m} / \mathrm{z}, 506.03\left(\mathrm{M}^{+}\right)$. Anal. calcd. for $\mathrm{C}_{26} \mathrm{H}_{20} \mathrm{ClN}_{3} \mathrm{O}_{2} \mathrm{~S}: \mathrm{C}, 61.71 ; \mathrm{H}, 03.98 ; \mathrm{N}, 08.30 ; \mathrm{S}, 12.67$; found: $\mathrm{C}, 61.69 ; \mathrm{H}$, $03.95 ; \mathrm{N}, 08.26 ; \mathrm{S}, 12.62 \%$.

4.7.2. 3-Chloro- $N$-\{4-[6-(4-chlorophenyl)-2-thioxo-1,2,5,6tetrahydropyrimidin-4-yl] phenyl\}-1-benzothiophene-2-carboxamide (8c)

IR $v\left(\mathrm{~cm}^{-1}\right): 3255(\mathrm{~N}-\mathrm{H}), 1650(\mathrm{C}=\mathrm{O}), 1435(\mathrm{C}=\mathrm{S}) ;{ }^{1} \mathrm{H}-\mathrm{NMR} \delta(\mathrm{ppm})$ : $10.08(\mathrm{~s}, 1 \mathrm{H}, \mathrm{NH}), 9.87(\mathrm{~s}, 1 \mathrm{H}, \mathrm{CONH}), 7.78-7.23(\mathrm{~m}, 12 \mathrm{H}, \mathrm{Ar}-\mathrm{H}), 4.03(\mathrm{~s}, 2 \mathrm{H}$, $\left.\mathrm{CH}_{2}\right) ;{ }^{13} \mathrm{C}-\mathrm{NMR} \delta(\mathrm{ppm}): 188,164.6,161.8,141.6,140.2,136.2,135.9,133.4$, 132.3, 129.9, 129.4, 128.6, 127.2, 126.7, 124.4, 124.3, 122.8, 121.7, 54.2, 41.7; MS: $\mathrm{m} / \mathrm{z}, 510.45\left(\mathrm{M}^{+}\right)$. Anal. calcd. for $\mathrm{C}_{25} \mathrm{H}_{17} \mathrm{Cl}_{2} \mathrm{~N}_{3} \mathrm{OS}$ : $\mathrm{C}, 58.82 ; \mathrm{H}, 03.36 ; \mathrm{N}$, 08.23; S, 15.56; found: C, 58.78; H, 03.32; N, 08.20; S, $15.51 \%$.

4.7.3.

3-Chloro- $N$-\{4-[6-(4-nitrophenyl)-2-thioxo-1,2,5,6tetrahydropyrimidin-4-yl]phenyl\}-1-benzothiophene-2-carboxamide (8d)

IR $v\left(\mathrm{~cm}^{-1}\right): 3242(\mathrm{~N}-\mathrm{H}), 1643(\mathrm{C}=\mathrm{O}), 1442(\mathrm{C}=\mathrm{S}) ;{ }^{1} \mathrm{H}-\mathrm{NMR} \delta(\mathrm{ppm})$ : $10.21(\mathrm{~s}, 1 \mathrm{H}, \mathrm{NH}), 10.00(\mathrm{~s}, 1 \mathrm{H}, \mathrm{CONH}), 8.34-7.65(\mathrm{~m}, 12 \mathrm{H}, \mathrm{Ar}-\mathrm{H}), 4.22(\mathrm{~s}$, $\left.2 \mathrm{H}, \mathrm{CH}_{2}\right) ;{ }^{13} \mathrm{C}-\mathrm{NMR} \delta(\mathrm{ppm}): 188,164.2,161.2,149.4,145.9,141.6,140.2$, $136.2,135.9,133.4,129.9,129.4,126.7,124.4,123.2,123.1,122.3,121.1$, 52.2, 41.2, 23.3, 22.1; MS: m/z, $521.01\left(\mathrm{M}^{+}\right)$. Anal. calcd. for $\mathrm{C}_{25} \mathrm{H}_{17} \mathrm{ClN}_{4} \mathrm{O}_{3} \mathrm{~S}_{2}$ : C, 57.63; H, 03.29; N, 10.75; S, 12.31; found: C, 57.59; H, 03.26; N, 10.70; $\mathrm{S}, 12.28 \%$.

\section{CONCLUSION}

In conclusion, a new series of benzothiophene substituted isoxazolines, pyrimidines and pyrazoles derivatives were synthesized and evaluated for their antibacterial and antifungal activities. The newly synthesized heterocyclics exhibited mordarate antibacterial activity against $S$. aureus, B. subtilis, $P$. aeruginosa and $E$. coli and significant antifungal activity against $C$. albicans, C. pannical, A. niger and R. oryzae. It can be concluded that these classes of compounds certainly holds great promise towards good active leads in medicinal chemistry. A further study to acquire more information concerning pharmacological activity is in progress.

\section{ACKNOWLEDGEMENTS}

This work was supported by the Higher Education Research Promotion and National Research University Project of Thailand, Office of the Higher Education Commission (Project No: EN1250B). The post doctoral fellowship grant from the Ratchadapisakesompote Endownment Fund, Chulalongkorn University (to G.N.) was gratefully acknowledged.

\section{REFERENCES}

[1] Mehta, K.J.; Patel, V.S.; Parikh, A.R. J. Indian. Chem. Soc. 1978, 50, 241-246.

[2] Mudaliar, V.; Joshi, V. Indian. J. Chem., Sect-B. 1995, 34B, 456-459.

[3] Hosni, G.; Saad, S.F. Acta. Chim. Acad. Sci. Hung. 1995, 86, 263-269.

[4] Hishmat, O.H.; El-Diwani H.I.; Melek, F.R. Indian. J. Chem., Sect-B. 1996, 35B, 30-35.

[5] Kuki Masakatsu, SakamotoYosuhiko, OtaYoichiro. Chem. Abstr. 1992, $117,131220 \mathrm{~d}$.

[6] Okabe, M.; Sun R.C.; Zenchoff, G.B. J. Org. Chem. 1991, 56, 439-449.

[7] Spada, R.; Klein R.S.; Otter, B.A. J. Heterocycl. Chem. 1989, 26, 18511857.

[8] Gilespie, R.J.; Giles, P.R.; Lerpiniere, J.; Dawson, C.E.; Bebbington, D. Adv. Heterocycl. Chem. 2005, 64, 1921-1927.

[9] Choi, H.S.; Wang, Z.; Richmond, W.; He, X.; Yang, K.; Jiang, T.; Sim, T.; Karanewsky, D.; Gu, X.J.; Zhou, V.; Liu, Y.; Ohmori, O.; Caldwell, J.; Gray N.; He, Y. Bioorg. Med. Chem. Lett. 2006, 16, 2173-2179.

[10] Robins, R.K.; Furcht, F.W.; Grauer, A.D.; Jones, J.W. J. Am. Chem. Soc. 1956, 78, 2418-2837.

[11] Holla, B.S.; Mahalinga, M.; Karthikeyan, M.S.; Akberali, P.M.; Shetty, N.S. Bioorg. Med. Chem. 2006, 14, 2040-2047.

[12] Yadav, L.S.; Dubey, S.; Yadav, L.S. Tetrahedron Lett. 2003, 59, 54115417.

[13] Kifli, N.; Clercq, E.D.; Balzarini, J.; Simons, C. Bioorg. Med. Chem. 2004, 12, 4245-4248.

[14] Humphries, A.C.; Gancia, E.; Gilligan, M.T.; Goodacre, S.; Hallett, D.; Merchant, K. J.; Thomas, S.R. Bioorg. Med.Chem. Lett. 2006, 16, 15181523.

[15] Taylor, E.C.; Carbon, J.A.; Hoff, D.R. J. Am. Chem. Soc. 1953, 79, 19041909.

[16] Wamhoff, H.; Wheling, B. Chem. Ber. 1975, 108, 2107-2110.

[17] Parkey S.; Castle, N. J. Heterocycl. Chem. 1986, 23, 1571-1579.

[18] British Pharmacopoeia. 2005, A300, Vol. IV, Appendix XIV.

[19] Vincent, J.G.; Vincent, H.W. Proc. Soc. Exp. Biol. Med. 1944, 55, 162169. 